\title{
Waterpipe Smoking among African American Muslims
}

\author{
Hammad Ali, Cynthia L. Arfken, PhD
}

Wayne State University, Detroit Michigan

Background: Traditionally used in the Middle East, Southeast Asia, and North Africa, the waterpipe has garnered popularity among adolescents and young adults worldwide. A common theme is that the countries with the highest percentage of waterpipe smoking are predominantly Muslim, despite Islamic ruling on tobacco, suggesting a cultural basis. A pilot study conducted by Abu-Ras et al. among American Muslims reported that religiosity and cultural practice were not leading factors for waterpipe smoking. Larger population-based data on prevalence and risk factors for waterpipe smoking among American Muslims are needed. The purpose of this study was to review the current medical literature for the prevalence between waterpipe smoking and African American Muslims.

Methods: Studies were electronically searched using the databases MEDLINE, PubMed, and academic search engine. The selected articles included cohort studies and cross sectional studies measuring the prevalence of waterpipe use in either general populations or a specific population of interest. Searches included combinations of the following keywords: "waterpipe smoking," "hookah," "African American," "race," "Muslim," and "culture."

Results: To date, studies have been conducted on African Muslims (Somali refugees) but it focused on cigarette use; and African American youth but it did not specify religious affiliation. The later study reported that hookah use did not differ significantly by gender between 2008 and 2010, however, prevalence rates increased significantly among African American students from 12.5\% in 2008 to $20.1 \%$ in 2010. Results suggest that African American youth as well as 
African Muslims are at risk for waterpipe smoking and that there is a common belief that waterpipe smoking is less harmful than cigarette smoking.

Conclusion: Further research on waterpipe smoking among indigent African American Muslims is needed. Ultimately, this information would be beneficial in identifying and understanding perceptions, progression, and trajectories of waterpipe smoking. This in turn will help in developing effective interventions to mitigate waterpipe smoking among an often-overlooked population. 